\title{
电化学石英晶体微天平研究 银的初始氧化过程*
}

黄义样 谢乃贤 周铁安 童定样

(湖南大学化学化工系,长沙 410082)

\section{关链词电化学石英晶体微天平、电化学策化银电极、性介质}

石英晶体微天平 QCM 应用于真空或气相实验作为灵敏的质量检测器已有较长时间, 但 直到 80 年代中期才将液相中的 QCM 同电化学方法联用而形成电化学石英晶体微天平 EQ$\mathrm{CM}^{[1-3]}$. $\mathrm{EQCM}$ 由压电石英晶片及适当设计的振荡电路构成, 在晶片两面沉积了金属膜电 极, 一个电极在气相, 另一个电极在溶液中作电解池工作电极. 对刚性沉积物, 晶体振荡频率 增量 $\Delta f$ 正比于工作电极上沉积物在单位面积 $A$ 上的质量改变 $\Delta m$. 只要 $\Delta f$ 小于晶体振 荡频率的百分之几, 溶剂的粘弹性不变且晶体工作于基频 $f_{0}$. 则有 Sauerbrey 方程成立 ${ }^{[4-6]}$

$$
\Delta f=-f_{0}^{i} \Delta m /(\rho N A),
$$

$N$ 取 $1.615 \times 10^{5} \mathrm{~cm} \cdot \mathrm{Hz}$ 是频率常数, $\rho$ 取 $2.648 \mathrm{~g} \cdot \mathrm{cm}^{-3}$ 是石英晶体密度. 因而当 $f_{0}$ 为 $9.25 \mathrm{MHz}$, 则 $\mathrm{EQCM}$ 质量灵敏度 $-\Delta m /(A \cdot \Delta f)=\rho N / f_{0}^{2}=5.0 \mathrm{ng} \cdot \mathrm{cm}^{-2} \cdot \mathrm{Hz}^{-1}$; 即若频率 精度为 $\mathrm{Hz}$ 级, 则可测纳克级质量变化.

EQCM 能在测电化学量(如电量、电流及电位等)的同时现场监测伴随电极过程的电极及 其表面涂层的质量变化. 目前国外研究十分活跃, 如用于研究金属电沉积、离子在聚合膜中流 动及其它界面现象 ${ }^{[6]}$. 然而它用于金属表面氧化层研究报道较少, 原因是该过程涉及金溃溶 解、吸附、氧化膜形成及表面变粗粘等影响 EQCM 响应的因素. 较早期的研究主要涉及表面 再构造现象如铁浴解 ${ }^{[7]}$ 、金、铜和银电极氧化时表面再构造; 后来才用于研究膜形成如 $\mathrm{Al}$ 电 极氧化成 $\mathrm{Al}_{2} \mathrm{O}_{3}$ 膜 ${ }^{[8]}$, 并证明 (1) 式仍然适用. 国内虽有综述文章 ${ }^{[6]}$, 但仍未见公开报道.

$\mathrm{Ag}$ 在 $\mathrm{KOH}$ 中电化学氧化虽有较多研究, 但目前对其初始氧化过程仍有三种不同观 点 ${ }^{[9]}$ : (1) 认为 $\mathrm{Ag}$ 仅以 $\mathrm{Ag}(\mathrm{OH})_{2}^{-}$形式络合溶解, (2) 认为形成沉积层, 其形式为 $\mathrm{Ag}_{2} \mathrm{O}$ 或 $\mathrm{AgOH}$ 尚无定论, (3) 认为络合溶解与形成沉积层都存在. 显然其中任一观点都涉及 $\mathrm{Ag}$ 电极及表面层质量改变, 而以往的研究均不能现场监测质量改变, 以致产生如上分歧. 本 文首次采用 $\mathrm{EQCM}$ 方法与恒电位、恒电流方法联用以阐明 $\mathrm{Ag}$ 在 $\mathrm{KOH}$ 中初始氧化过程.

\section{1 实验}

\section{1 仪器}

HDV-7 晶体管恒电位仪完成恒电流及恒电位，DT-890 数字多用表测电压，CN3165

* 湖南省自然科学基金资助项目. 
高分辨率频率计数器(中国台湾)测频率, 微安表测电流, 秒表计时.

\section{2 药品}

$\mathrm{KOH}$ (A. R. 级) 用二次水配成 $0.7219 \mathrm{~mol} \cdot \mathrm{dm}^{-3}$ 及 $7.051 \mathrm{~mol} \cdot \mathrm{dm}^{-3}$ 溶液. 实验前通氮 除氧约 $15 \mathrm{~min}$.

\section{3 电极}

EQCM 置于三电, 极电解池中 ${ }^{[10]}$ 完成恒电位及恒电流测量. 辅助电极为大面积螺旋状银 丝; 参比电极为 $\mathrm{Hg} / \mathrm{HgO}(\mathrm{s}), 7.051 \mathrm{~mol} \cdot \mathrm{dm}^{-3} \mathrm{KOH}$ (本文所有电位 $E$ 均为相对该电极的值); 工作电极为 $9.3 \mathrm{MHz}$ 的 $\mathrm{AT}$ 切型石英晶片上真空沉积的 $\mathrm{Ag}$ 电极, 实验前需在氯化镀液中 铰一层 $\mathrm{Ag}$ 以防损耗, 除特殊说明外, 电极面积 $0.28 \mathrm{~cm}^{2}$.

\section{$1.4 \mathrm{EQCM}$ 振荡电路及装置框图}

如图 1 所示, 该舁路对文献 [8] 的电路进行改进, 更为简单易行, 调节电位器 P 改变反馈 系数易于在溶液中起振. 频率稳定度为 $\sim \pm 2 \mathrm{~Hz} \cdot \mathrm{min}^{-1}$.

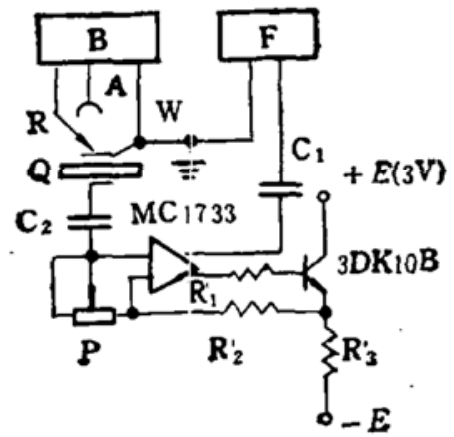

图 1 振荡电路及装置框图 $A$ 为辅助电极, $W$ 为工作电伋, $R$ 为参比电极, $Q$ 为石英晶片, $P$ 为多圈电䎲器, $C_{1}$ 及 $C_{2}$ 为电 容, $R_{1}$ 至 $R_{3}$ 为电阻, $B$ 为栢电位仪, $F$ 为频率计

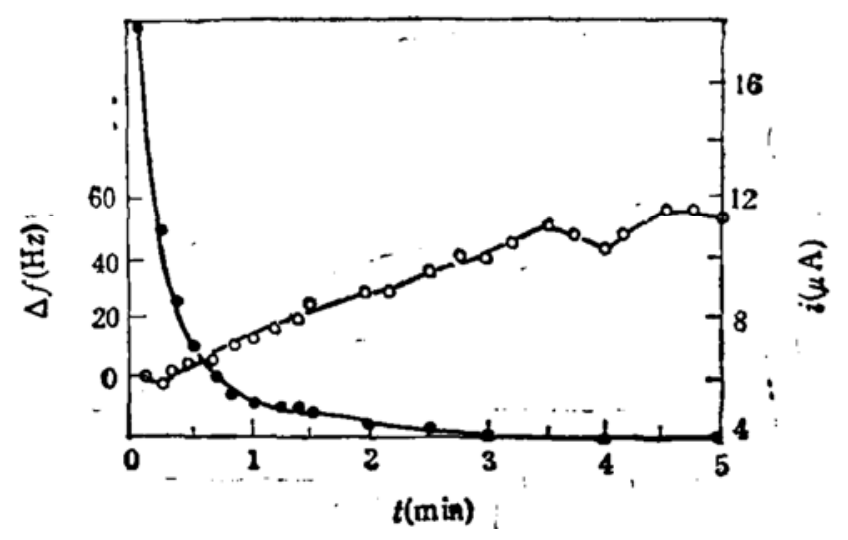

图2 恒电位 $E=0.300 \mathrm{~V}, 302 \mathrm{~K}, 0.7219 \mathrm{~mol} \cdot \mathrm{dm}^{-3} \mathrm{KOH}$ 0 为 $\Delta f$ 与 $\boldsymbol{t}$ 关系, $\bullet$ 为 $i$ 与 $\boldsymbol{t}$ 关系

\section{2 结果讨论}

\subsection{Sauerbrey 方程验证}

恒电流于 $20 \mu \mathrm{A}$, 在 $A$ 为 $0.32 \mathrm{~cm}^{2}$ 的电极上实验, 得电铰时间 $t$ 与频率变化 $\Delta f$ 之线性 关系为 $\Delta f / t--8.00 \times 10^{2} \mathrm{~Hz} \cdot \mathrm{min}^{-1}$. 恒电流则时间正比于电量, 电流效率百分之百, 则电 量正比于被沉积 $\mathrm{Ag}$ 的质量 $\Delta m$, 上述线性关系说明 $\Delta f$ 与 $\Delta m$ 成正比例. 由电量求出 $\Delta m$ 后, 得实测质量灵敏度 $-\Delta m /(A \cdot \Delta f)-5.2 \pm 0.5 \mathrm{ng} \cdot \mathrm{cm}^{-2} \cdot \mathrm{Hz}^{-1}$, 与方程 (1) 计算的 $5.0 \mathrm{ng} \cdot \mathrm{cm}^{-2} \cdot \mathrm{Hz}^{-1}$ 相吻合 $\left(f_{0}-9.25 \mathrm{MHz}\right)$. 恒电流于 $40 \mu \mathrm{A}$, 亦证实该结论.

\section{$2.2 \mathrm{Ag}$ 溶解的证据}

初始氧化过程系指较小电位范围 (如小于 $0.35 \mathrm{~V}$ 左右) 银的氧化过程. 图 2 是恒电位 $0.300 \mathrm{~V}$ 的结果, 随时间增加, 电流 $i$ 下降而 $\Delta f$ 增大, 表明银溶解引起质量变小占优势. 若 只有银的溶解, 则每摩尔电子的电量将引起 $108 \mathrm{~g}$ 银的溶解即 $\frac{\mathrm{d} m}{\mathrm{~d} n}--108 \mathrm{~g} \cdot \mathrm{mol}^{-1}$ （ $n$ 是通 
过电极的电子摩尔数). 据 Faraday 定律及式 (1), 可由图 2 求得 $\frac{\mathrm{d} m}{\mathrm{~d} n}$ 与时间关系, 显示 $\frac{\mathrm{d} m}{\mathrm{~d} n}$ 大体上为负值, 最小值为 $-18 \mathrm{~g} \cdot \mathrm{mol}^{-1}$, 与 $-108 \mathrm{~g} \cdot \mathrm{mol}^{-1}$ 相差甚远. 表明在 $0.300 \mathrm{~V}$ 下 银的阳极行为决非一个单纯的溶解过程, 而是伴随着某个质量增加过程. 后一过程的存在, 在 恒电流法中将更易体现.

\section{$2.3 \mathrm{AgOH}$ 沉积层、 $\mathrm{OH}^{-}$吸附与 $\mathrm{Ag}$ 溶解过程}

图 3a 是 $15 \mu \mathrm{A}$ 电流下恒电流电解曲线, 由 $\mathrm{EQCM}$ 测得 $\Delta f$ 与 $\boldsymbol{t}$ 曲线也示于图中, 它 们显示了电解过程中电极质量改变. 据式 (1) 及 Faraday 定律易将图 3a 转化为图 $3 \mathrm{~b}$. 从 图 $3 \mathrm{~b}$ 可看到 $\frac{\mathrm{d} m}{\mathrm{~d} n}$ 随 $t$ 或 $\frac{n}{A}$ 的三个变化阶段: (1) 开始阶段 $\frac{\mathrm{d} m}{\mathrm{~d} n}$ 为负并下降至一负峰值;
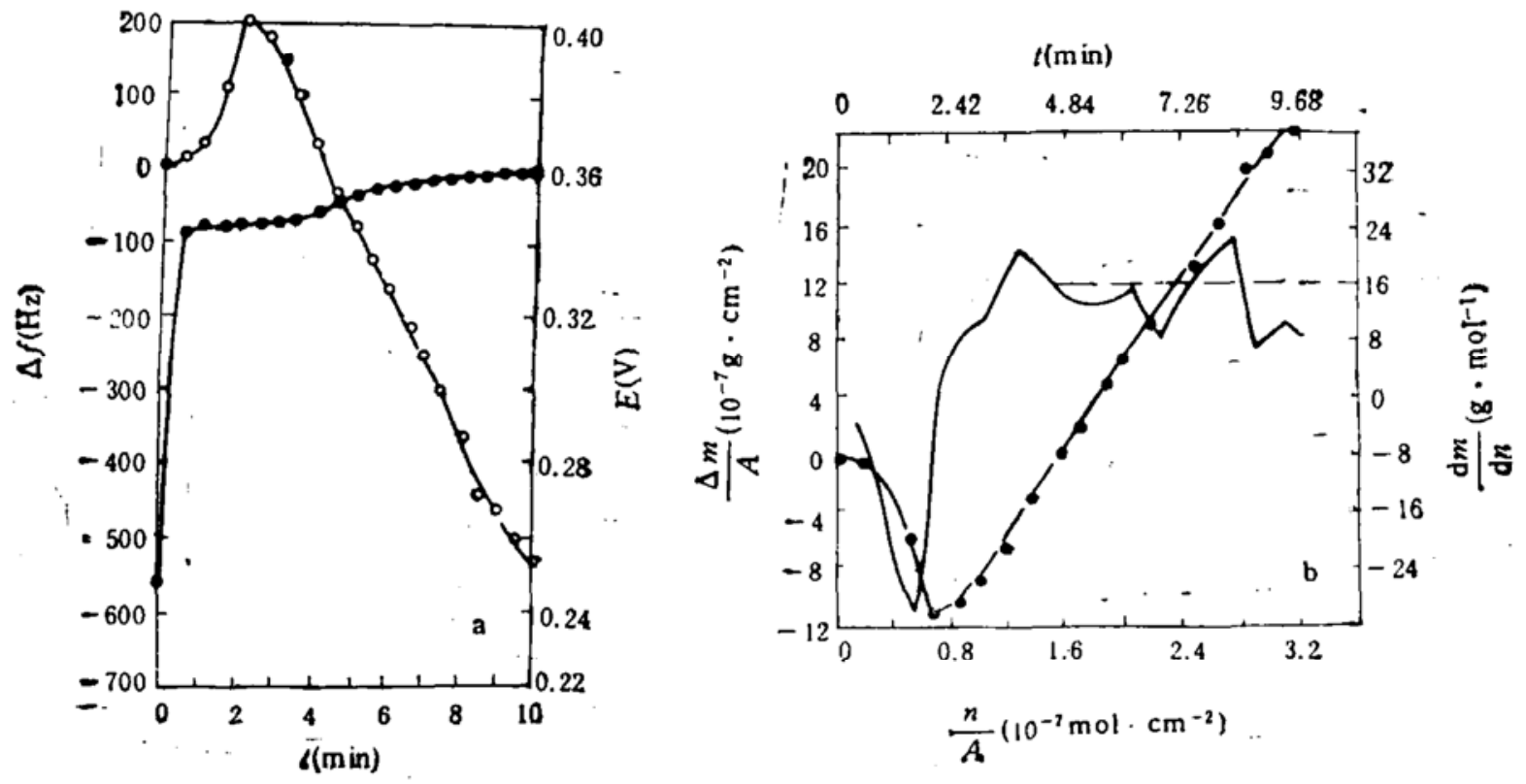

图 3 恒电流 $15.0 \mu \mathrm{A}$ 氧化, $0.7219\left(\mathrm{~mol} \cdot \mathrm{dm}^{-3}\right) \mathrm{KOH}, 300 \mathrm{~K}$

a. 为 $\Delta f$ 与 8 关系 $(0), E$ 与 $t$ 关系 $(\bullet)$;

b. 为 $\Delta m / A$ 与 $n / A$ 或 $t$ 关系 $(\bullet), \mathrm{d} m / \mathrm{d} n$ 与 $n / A$ 或 $t$ 关系(折线)

（2）中间阶段 $\frac{\mathrm{d} m}{\mathrm{~d} n}$ 上升并由负转变为正值, 达到一正峰值; (3) 后阶段 $\frac{\mathrm{d} m}{\mathrm{~d} n}$ 缓慢下降, 然后虽 有波动但较平缓. 恒电流为 20 及 $25 \mu \mathrm{A}$ 也显示类似规律. 各电流下, 后阶段中一个较大的 $\frac{n}{A}$ 范围 ${ }^{\mathrm{s}}$ 内, $\frac{\mathrm{d} m}{\mathrm{~d} n}$ 平均值为 $16 \pm 1 \mathrm{~g} \cdot \mathrm{mol}^{-1}$. 各电流下, 正负峰值参数列于表 1 .

假若银电极的阳极行为是单纯的银溶解则 $\frac{\mathrm{d} m}{\mathrm{~d} n}=-108 \mathrm{~g} \cdot \mathrm{mol}^{-1}$, 若形成 $\mathrm{AgOH}$ 沉积层则 $\frac{\mathrm{d} m}{\mathrm{~d} n}=17 \mathrm{~g} \cdot \mathrm{mol}^{-1}$, 若形成 $\mathrm{AgOH}$ 沉积层并有 $\mathrm{OH}^{-}$的 $1: 1$ 吸附则 $\frac{\mathrm{d} m}{\mathrm{~d} n}-34 \mathrm{~g} \cdot \mathrm{mol}^{-1}$, 若嫄成 $\mathrm{Ag}_{2} \mathrm{O}$ 沉积层则 $\frac{\mathrm{d} m}{\mathrm{~d} n}-8 \mathrm{~g} \cdot \mathrm{mol}^{-1}$. 开始阶段 $\frac{\mathrm{d} m}{\mathrm{~d} n}$ 为负值并下降表明银溶解过程存在及加强, 但负峰值为 -20 至 $-31 \mathrm{~g} \cdot \mathrm{mol}^{-1}$ (表 1 ), 明显大于 $-108 \mathrm{~g} \cdot \mathrm{mol}^{-1}$, 显然在此过程中伴随着

1) $\frac{n}{A}$ 范围为 $1.32-2.64(i=15 \mu \mathrm{A}), 1.54-2.62(i=20 \mu \mathrm{A})$ 以及 $1.10-2.47(i=25 \mu \mathrm{A})\left(10^{-7} \mathrm{~mol}^{-\mathrm{cm}^{-2}}\right)$. 
表 1 各恒电流 $i$ 下, $\frac{\mathrm{d} m}{\mathrm{~d} n}, \frac{n}{A}, E$ 与 $t$ 关系”

\begin{tabular}{|c|c|c|c|c|c|c|}
\hline$l(\mu \mathrm{A})$ & \multicolumn{2}{|c|}{15.0} & \multicolumn{2}{|c|}{20.0} & \multicolumn{2}{|c|}{25.0} \\
\hline 峰型 & 负略 & 正蜂 & 负峰 & 正峰 & 负盽 & 正峰 \\
\hline$\frac{\mathrm{d} m}{\mathrm{~d} n}\left(\mathrm{~g} \cdot \mathrm{mol}^{-1}\right)$ & -31 & 22 & -30 & 35 & -20 & 17 \\
\hline$\frac{n}{A}\left(10^{-y} \mathrm{~mol} \cdot \mathrm{cm}^{-2}\right)$ & 0.59 & 1.3 & 0.12 & 0.16 & 0.14 & 1.02 \\
\hline$b(8)$ & 109 & 226 & 10.0 & 22.0 & 13.0 & 110 \\
\hline$E(\mathrm{~V})$ & 0.342 & 0.346 & 0.352 & 0.50 & 0.333 & 0.363 \\
\hline
\end{tabular}

a) 条件同图 3 .

沉积过程. 中间阶段 $\frac{\mathrm{d} m}{\mathrm{~d} n}$ 上升表明沉积速度超过溶解速度,达到正峰值约在 17 至 $35 \mathrm{~g} \cdot \mathrm{mol}^{-1}$ （表 1), 表明所有 $\mathrm{Ag}(\mathrm{I})$ 全部转化为 $\mathrm{AgOH}$, 并可能表面有 $\mathrm{OH}^{-}$吸附存在. 后阶段,在 $\frac{n}{A}$ 值的一个比较大的范围内 $\frac{\mathrm{d} m}{\mathrm{~d} n}$ 平均值为 $16 \pm 1 \mathrm{~g} \cdot \mathrm{mol}^{-1}$ 或 $\frac{\mathrm{d} m}{\mathrm{~d} n}$ 在 $16 \mathrm{~g} \cdot \mathrm{mol}^{-1}$ 波动(参见图 $3 \mathrm{~b}$ 的虚线), 说明沉积层不是 $\mathrm{Ag}_{2} \mathrm{O}$ 而是 $\mathrm{AgOH}$ 为主, 同时伴随有沉积层表面对 $\mathrm{OH}^{-}$的吸附 及脱附。

\section{3 结 论}

（1）本文报道了”在我们实验室中实现了 EQCM 技术,并对文献报道电路进行了改进,使 之简便易行, 质量灵敏度达 $5.0 \mathrm{ng} \cdot \mathrm{cm}^{-2} \cdot \mathrm{Hz}^{-1}$, 可感知亚单分子层之质量变化. 这一技术将 会在电化学、电分析及金属腐蚀等界面科学领域找到极为广泛的应用.

(2) 将 EQCM 技术和恒电位、恒电流方法联用, 实现了对银在 $\mathrm{KOH}$ 中初始氧化过程的 电极质量变化的首次现场监测. 证实了该体系阳极行为中溶解与沉积过程的存在, 而沉积物 最初形式是 $\mathrm{AgOH}$ 及对 $\mathrm{OH}^{-}$的吸附层, 不是 $\mathrm{Ag}_{2} \mathrm{O}$.

\section{参考文献}

[1] Bruckenstein, S., Shay, M., J. Electroaral. Chem. 1985, 188: 131-136.

[2] Bruckenstein, S., Shay, M., Electrochimica Acta, 1985, 30: 1295-1300.

[3] Kaufmann, J. H., Kanazawa, K. K., Street, G. B., Phys. Rev. Lett., 1984, 53: 2461-2464.

[4] Sautrbrey, G. Z., Z. Phys., 1959, 155:206.

[5] Kanazawa, K. K., Gordon Il, J. G., Anal.Chem., 1985. 59:1770.

[6] 朱则善,化学通报, 1991, (9): 27-31.

[7] Hager, H. E., Ruedisveli, R. D., Buehler, M. E., Corrosion. 1986, 42:345.

[8] Deakin, M. R., Melroy, O. R., J. Electrochem. Soc., 1989, 136: 349-352.

[9] Hapel. M., Tomkiewicz, M., J. Electrochem. Soc., 1984, 131: 1288-1294.

[10] Bourkane, S. et al., Electrochimica Acta, 1988, 34:1081-1092. 